\title{
Correlación entre las estrategias SITA Estándar 24 -2 y SITA Faster 24 -2 para evaluar el daño campimétrico en pacientes con Glaucoma Primario de Ángulo Abierto
}

\section{Correlation between the SITA Standard 24-2 and SITA Faster 24-2 strategies to assess campimetric damage in patients with Primary Open Angle Glaucoma.}

\author{
Justo Manuel Pavía', Guillermo Raúl Vera-Duarte $\mathbb{D}^{1}$, Martín Fernando Arrúa-Caballero ${ }^{1}$
}

${ }^{1}$ Universidad Nacional de Asunción, Facultad de Ciencias Médicas, San Lorenzo, Paraguay.

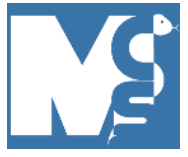

Recibido: 08/07/2021

Revisado: 11/08/2021

Aceptado:30/08/2021

\section{Autor correspondiente}

Guillermo Raúl Vera Duarte Universidad Nacional de Asunción, San Lorenzo, Paraguay guillermoveraduarte@gmail.com

\section{Conflictos de interés}

Los autores declaran no poseer conflictos de interés.

\section{Fuente de financiación}

Los autores no recibieron apoyo financiero para la investigación, autoría y/o publicación de este artículo.

Este artículo es publicado bajo una licencia de Creative Commons Reconocimiento 4.0 Internacional.

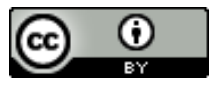

\section{RESUMEN}

Introducción: Las estrategias de SITA Estándar fueron aprovechadas por más de dos décadas, pero buscando aun mejorar el tiempo sin perder la calidad, se volvió a crear una nueva estrategia más corta, a la cual llamaron estrategia FASTER. Objetivo: Determinar la correlación entre las estrategias SITA Estándar 24 -2 y SITA Faster 24 -2 para evaluar el daño campimétrico en pacientes con Glaucoma Primario de Angulo Abierto. Metodología: Estudio observacional analítico. RESULTADOS: Se analizó 74 resultados de análisis del campo visual utilizando dos técnicas SITA Estándar $24-2$ y SITA Faster 24 - 2. El tiempo de prueba para la estrategia SITA Estándar fue de 379,64 $\pm 72,75$ segundos y para la estrategia SITA Faster 170,93 $\pm 49,17(p<0,0001)$, probando una reducción de tiempo del $55 \%$ para la segunda. Los valores de desviación media, desviación patrón estándar y el índice de campo visual no mostraron diferencias estadísticamente significativas entre ambas pruebas. La Prueba de Hemicampo de glaucoma con el cálculo del índice Kappa de Cohen arroja un valor de 0,316 con un intervalo de confianza del $95 \%$ de $(0,098-0,534)$ lo que indica que es estadísticamente significativo. Discusión: Los resultados generales indican que es posible reemplazar la estrategia SITA Estándar 24 -2 con la estrategia SITA Faster 24 - 2. Este cambio será especialmente útil para aumentar la frecuencia de exámenes campimétricos, determinante para una detección más temprana de la progresión del glaucoma y así mejorar las conductas terapéuticas que se toman con los pacientes que acuden a nuestro servicio.

Palabras clave: Campimetría; SITA Estándar; SITA Faster; correlación; Glaucoma Primario de Ángulo Abierto.

\section{ABSTRACT}

Introduction: SITA Standard strategies were used for more than two decades, but still seeking to improve time without losing quality, a new shorter strategy was created again, which they called the FASTER strategy. Objective: To determine the correlation between the SITA Standard 24-2 and SITA Faster 24-2 strategies to evaluate the visual field damage in patients with Primary Open Angle Glaucoma. Methodology: Analytical observational study. Results: 74 visual field analysis results were analyzed using two techniques SITA Standard $24-2$ and SITA Faster 24 - 2. The test time for the SITA Standard strategy was $379.64 \pm 72.75$ seconds and for the SITA strategy Faster $170.93 \pm 49.17(p<0.0001)$, proving a $55 \%$ time reduction for the second. The mean deviation, standard deviation and visual field index values do not show statistically significant differences between both tests. The Glaucoma Hemifield Test with the calculation of the Cohen's Kappa index gives a value of 0.316 with a $95 \%$ confidence interval of $(0.098-0.534)$ which indicates that it is statistically significant. Discussion: The overall results indicate that it is possible to replace the SITA Standard 24-2 strategy with the SITA Faster 24-2 strategy. This change will be especially useful to increase the frequency of campimetric examinations. A greater frequency of examinations is important for earlier detection of glaucoma progression and thus improve the therapeutic behaviors that are taken with the patients who come to our service. 


\section{INTRODUCCIÓN}

El Glaucoma primario de ángulo abierto (GPAA) se define como una neuropatía óptica crónica lentamente progresiva, crónica, con cambios característicos de daño de la papila del nervio óptico y pérdida del campo visual (1), acompañado o no de hipertensión ocular. El GPAA, también conocido como glaucoma crónico de angula abierto o glaucoma crónico simple, es una enfermedad bilateral, pero asimétrica, de inicio insidioso e indoloro. Los pacientes son asintomáticos en su mayoría, hasta estados avanzados de la enfermedad. El diagnóstico de GPAA se basa en las alteraciones de la cabeza del nervio óptico, en la capa de fibras nerviosas y en los resultados de evaluaciones del campo visual (1). Una evaluación oftalmológica completa, incluyendo la del nervio óptico y una campimetría del paciente en seguimiento, es esencial para el manejo adecuado del glaucoma (2).

En cuanto a la campimetría ideal debería idealmente probar todas las intensidades en todas las localizaciones, sin embargo, se ha visto que no es muy práctico realizar pruebas con las distintas intensidades $\mathrm{y}$, en ocasiones, ni siquiera es posible por las limitaciones que implica en duración de la prueba. La duración de las primeras pruebas de umbral oscilaba en promedio entre los 12 y 20 minutos por ojo. Esta duración es agotante para los pacientes y limita el número de pruebas que se pueden ejecutar (3), dato no menor para hospitales del sistema de salud pública con gran afluencia de pacientes.

A modo de mejorar esta limitación, a finales de los años 80 , se comenzaron a desarrollar estrategias de campimetría conocidas como SITA (Swedish In-teractive Treshold Algorithm) (3), cuyo fin era la de disminuir el tiempo duración de la prueba con la posibilidad de mantener la calidad del estudio. Las estrategias SITA se compararon con las estrategias originales y se encontró que se redujeron los tiempos de estudio a la mitad, sin empeorar la variabilidad entre las pruebas (4). En cuanto a los valores umbrales en las nuevas estrategias aumentaron con respecto a las pruebas más largas, en las cuales el umbral tendía a disminuir debido al cansancio y desconcentración del paciente (5). A pesar de la reducción en el tiempo, la detección de pérdidas por glaucoma se mantuvo similar a los algoritmos previos $y$, por lo tanto, obtuvieron gran aceptación $(6,7)$.

Estas estrategias de SITA fueron aprovechadas por más de dos décadas, pero buscando aun mejorar el tiempo sin perder la calidad, se volvió a crear una nueva estrategia más corta, a la cual llamaron estrategia FASTER. Esta estrategia, FASTER presenta como principales características seis variaciones básicas con respecto a las demás estrategias, dichas diferencias se remontan a: la intensidad de la que parte de los puntos primarios, que se encuentran en el centro de cada cuadrante del campo visual de 24 grados, era de $25 \mathrm{~dB}$, en el FASTER la secuencia de prueba comienza en el nivel de umbral normal corregido por la edad, por lo que se reduce el número de presentaciones de estímulo para la mayoría de los ojos. Los cruces de umbral: se realizaban dos secuencias de escalón para encontrar el umbral en los puntos primarios, mientras que en FASTER, solo se cruza una vez. Modelos de probabilidad previa: Las primeras estrategias SITA tuvieron que modelar su probabilidad de acuerdo a las pruebas de umbral completo a fin de poder detenerse cuando existía una confiabilidad adecuada. Sin embargo, la estrategia FASTER usa los datos obtenidos de las pruebas FAST normales para tener nuevos estimados y datos que logran agilizar la prueba. No vuelve a probar los puntos cero: La estrategia FASTER no vuelve a enviar estímulos a los puntos donde no hubo sensibilidad al proyectar 10000 apostilbs. Índices de confiabilidad: Los falsos negativos dejan de probarse. Las pérdidas de fijación con método Heijl Krakau (enviando estímulos a la mancha ciega) dejan de probarse de esa manera, ya que existe un monitor infrarrojo de la mirada y, en ocasiones, se responde a estímulos enviados a la mancha ciega sin que sea tenga que ver con la confiabilidad de un estudio $(8,9)$. Intervalos entre estímulos: en los cuales existía un lapso de 300 milisegundos en las estrategias anteriores, en cambio en la estrategia FASTER ya fue eliminado este retraso.

Todas estas diferencias citadas, ha hecho a considerar, que, de realizarse correctamente, la estrategia FASTER permite obtener mayor número de pruebas, por consecuente mayor recaudación de datos, en un paciente que se encuentra en seguimiento del daño glaucomatoso a fin de lograr un mejor control y disminución de progresión de la enfermedad.

\section{METODOLOGÍA}

Se realizó un estudio observacional analítico, en la que se evaluaron 74 resultados de análisis del campo visual utilizando dos técnicas campimetrícas de SITA Estándar $24-2$ y SITA Faster $24-2$ en pacientes con Glaucoma Primario de Ángulo Abierto que acudieron a realizarse pruebas de campimetría en el servicio de Oftalmología del Hospital de Clínicas de la Universidad Nacional de Asunción de junio a noviembre del 2019.

El objetivo fue determinar la correlación entre ambas estrategias para evaluar el daño campimétrico en pacientes con GPAA. Se incluyeron pacientes de ambos 
sexos, mayores de 18 años, con diagnóstico de GPPA que cumplían los criterios de la guía de buena práctica médica de la Academia Americana de Oftalmología, alfabetizados, con AV igual o mejor a 20/200 con corrección. Se excluyeron pacientes menores de 18 años, con alteraciones retinianas maculares, con alteraciones severas de los medios transparentes del ojo, con defecto refractivo mayor a +/-6.00 D de esfera $y+/-4.00 \mathrm{D}$ de cilindro, pacientes con diagnóstico de ojo severo o enfermedad de superficie ocular asociada, así como aquellos que no hayan terminado el ciclo primario de la educación básica.

Se evaluó la Desviación Media (MD), Desviación Estándar Patrón (PSD), Prueba de Hemicampo para Glaucoma (PHG) e Índice Campo Visual (VFI), así como el tiempo empleado e Índices de confiabilidad con las diferentes estrategias (SITA Estándar $24-2$ y SITA Faster 24 - 2) en pacientes con GPAA. También se evaluó la satisfacción del paciente en relación al tipo de estrategia utilizada en la evaluación del daño campimétrico.

\section{RESULTADOS}

Fueron realizadas en total 84 evaluaciones campimétricas con las dos estrategias, 13 resultados fueron descartados por no cumplir criterios de confiabilidad, resultando en un total de 74 campos visuales como parte del estudio. De los 74 resultados de análisis del campo visual utilizando dos técnicas SITA Estándar 24-2 y SITA Faster 24 - 2 con el fin de estudiar la concordancia entre los resultados entre ambas pruebas. Los resultados cuantitativos se presentan en la Tabla 1, junto con las pruebas de significación estadística del tiempo, Índice del Campo Visual, Desviación Media, Desviación Estándar Patrón y Falsos Positivos.

De la Tabla 1 se desprende que no existen diferencias significativas entre las medias de MD, PSD y VFI, por lo que puede decirse con un nivel de confianza del $95 \%$ que los valores medios de MD, PSD y VFI tomados con ambos métodos no son significativamente diferentes. Se establecieron los coeficientes de correlación para el VFI, DM, PSD y los Falsos Positivos entre ambas estrategias. (Figuras 1,2 3 y 4).

TABLA 1. COMPARACIÓN DE LOS RESULTADOS CUANTITATIVOS CON SITA ESTÁNDAR 24 - 2 Y SITA FASTER 24 - 2 (N=74).

\begin{tabular}{|l|l|l|l|}
\hline Variable & SITA Estándar 24 -2 (media \pm SD) & SITA Faster 24 - 2 (media \pm SD) & p valor \\
\hline Tiempo (segundos) & $379,64 \pm 72,75$ & $170,93 \pm 49,17$ & $p<0,0001$ \\
VFI & $81,84 \pm 24,85$ & $84,38 \pm 20,97$ & 0,025 \\
MD & $-6,88 \pm 8,74$ & $-6,58 \pm 7,14$ & 0,523 \\
PSD & $4,91 \pm 3,71$ & $5,18 \pm 4,79$ & 0,339 \\
Falsos positivos & $3,64 \pm 8,54$ & $8,39 \pm 13,01$ & 0,000056 \\
\hline
\end{tabular}

FIGURA 1: CORRELACIÓN PARA VFI ENTRE LAS ESTRATEGIAS SITA ESTÁNDAR 24 - 2 Y SITA FASTER 24 -2.

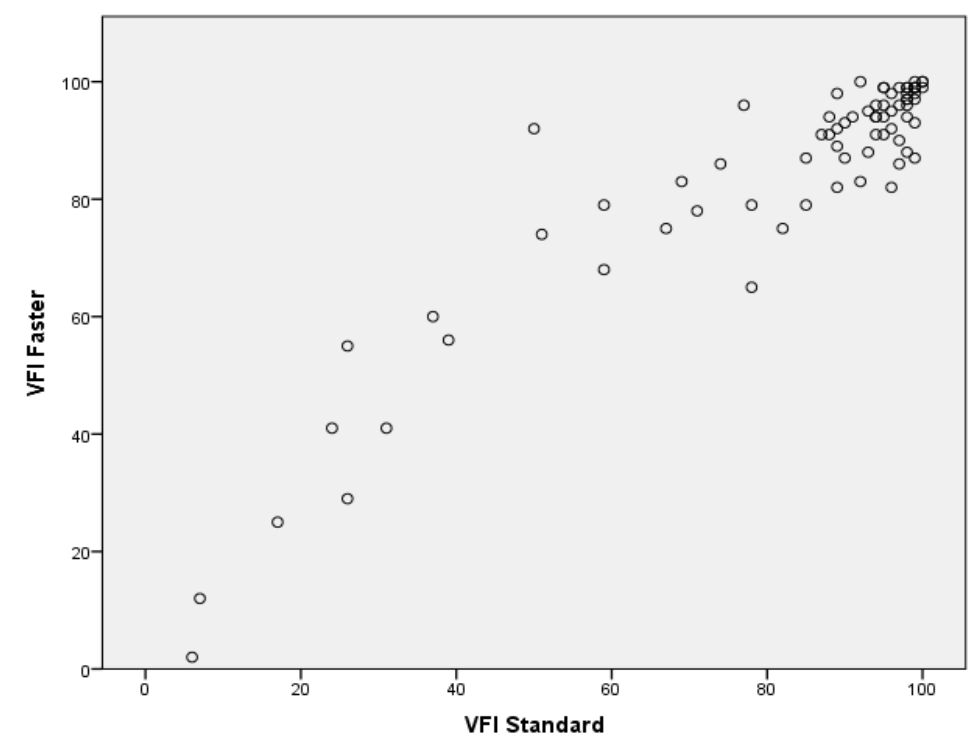


FIGURA 2: CORRELACIÓN PARA MD STANDARD ENTRE LAS ESTRATEGIAS SITA ESTÁNDAR 24 - 2 Y SITA FASTER 24 -2.

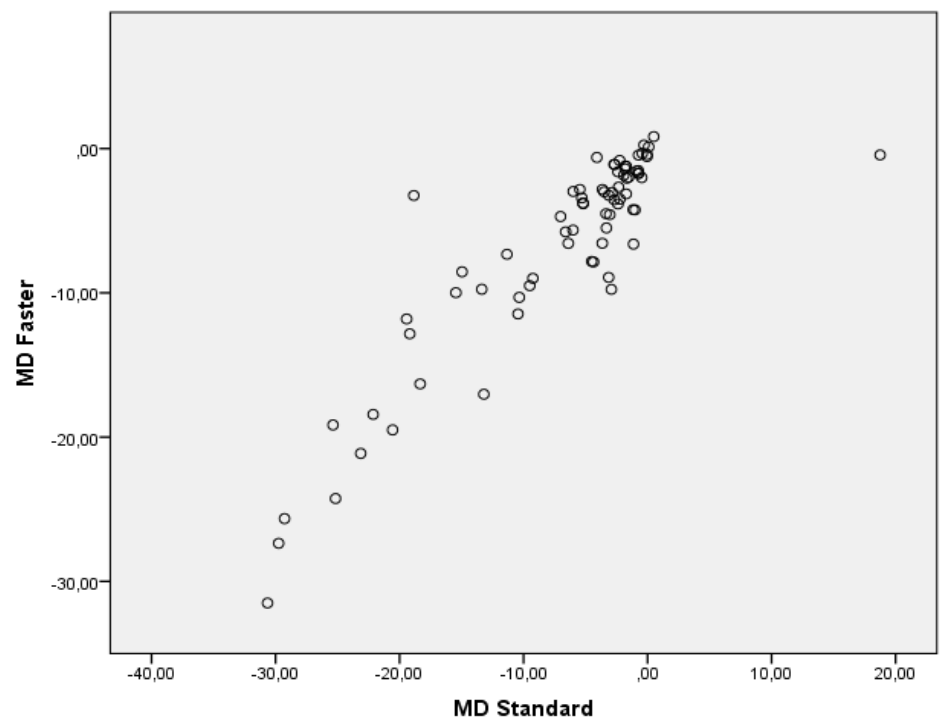

FIGURA 3: CORRELACIÓN PARA PSD STANDARD ENTRE LAS ESTRATEGIAS SITA ESTÁNDAR 24 - 2 Y SITA FASTER 24 -2.

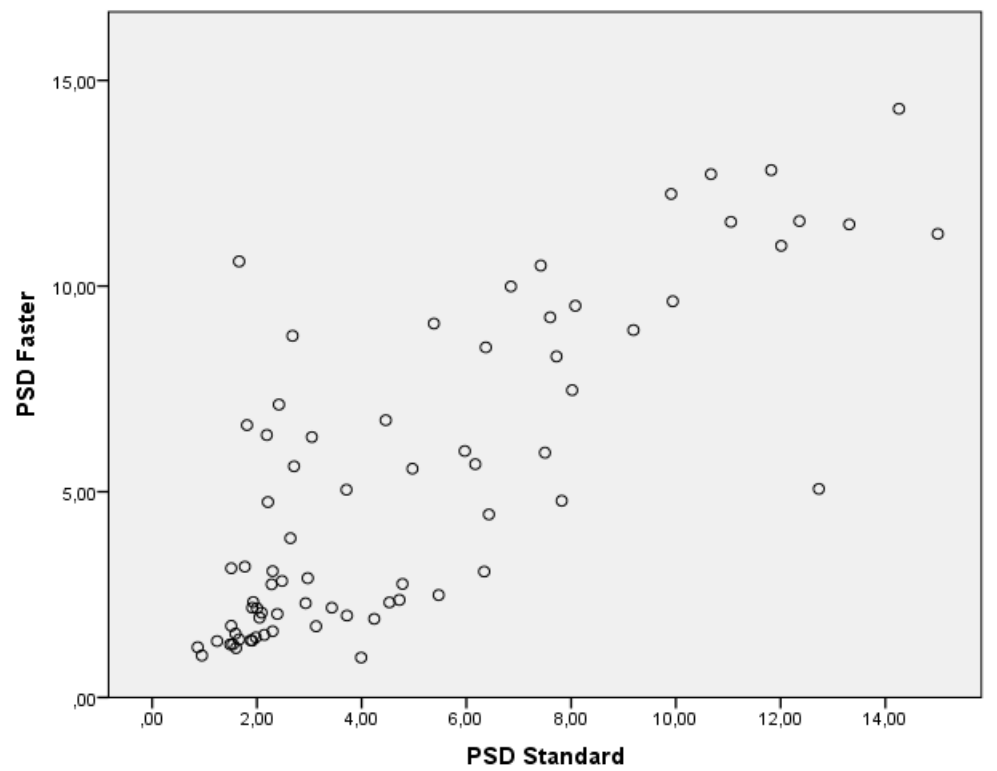

FIGURA 4: CORRELACIÓN PARA FALSOS POSITIVOS ENTRE LAS ESTRATEGIAS SITA ESTÁNDAR 24 - 2 Y SITA FASTER 24 -2.

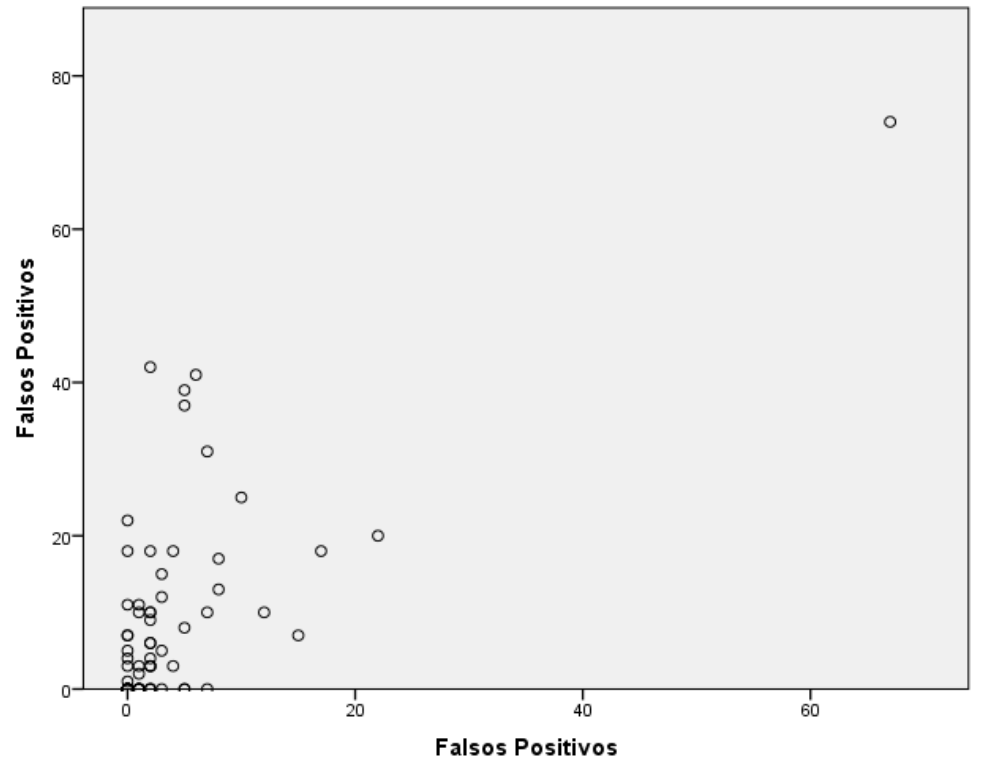


En la Tabla 2 se observa la concordancia entre los resultados en la prueba de hemicampo para glaucoma (PHG), comparando ambos métodos. El cálculo del índice Kappa de Cohen (10) arroja un valor de 0,316 con un intervalo de confianza del $95 \%$ de $(0,098-$ $0,534)$ lo que indica que es estadísticamente significativo. El valor de 0,316 se considera una concordancia discreta entre ambas pruebas. De la tabla también se desprende que la mayor proporción de concordancia se encuentra en el resultado "fuera de los límites normales". Los pacientes no manifestaron ninguna diferencia subjetiva entre las diferentes estrategias y se mostraron más satisfechos con la Estrategia SITA Faster 24 - 2 por el menor tiempo de estudio.

\section{TABLA 2. CONCORDANCIA ENTRE LOS RESULTADOS EN LA PRUEBA DE HEMICAMPO PARA GLAUCOMA UTILIZANDO SITA ESTÁNDAR 24 - 2 Y SITA FASTER 24 - 2 ( $\mathrm{N}=74)$}

\begin{tabular}{|c|c|c|c|c|c|}
\hline \multicolumn{6}{|c|}{ SITA Estándar 24 - 2} \\
\hline & & DLN & FLN & CD & SAA \\
\hline & DLN & 7 & 1 & 5 & 0 \\
\hline $\begin{array}{l}\text { N } \\
\text { I } \\
\text { d }\end{array}$ & FLN & 6 & 41 & 3 & 1 \\
\hline$\frac{\vec{d}}{\tilde{w}}$ & CD & 1 & 7 & 1 & 0 \\
\hline 岕 & SAA & 0 & 0 & 1 & 0 \\
\hline
\end{tabular}

DLN: dentro del límite normal, FLN: fuera del límite normal, CD: caso dudoso, SAA: sensibilidad anormalmente alta.

\section{DISCUSIÓN}

Este estudio clínico demostró una reducción significativa del tiempo de estudio comparando la estrategia SITA FASTER (DM 170,93 \pm 49,17) comparada con la estrategia SITA ESTÁNDAR (DM $379,64 \pm 72,75)$. El resultado con la estrategia SITA Faster fue $55 \%$ más rápido en concordancia con lo obtenido por otros investigadores que reportaron un tiempo en segundos de $171,9(45,3)$ para SITA Faster y $369,5(64,5)$ para SITA Estándar ( $p<0.001)$, con un tiempo 53,5 \% más veloz para la estrategia SITA Faster (3). El tiempo de estudio depende considerablemente el grado de perdida de campo visual por el GPAA. Los pacientes con $\mathrm{VFI}<50 \%$ obtuvieron hasta $40 \%$ más de tiempo de estudio, comparable con lo obtenido por otros investigadores que encontraron el doble de tiempo de estudio (3).

No existen diferencias significativas entre las medias de MD, PSD y VFI por lo que puede decirse con un nivel de confianza del $95 \%$ que los valores medios de MD y PSD y VFI tomados con ambos métodos no son significativamente diferentes. El Resultado es comparable a los datos obtenidos por otras investigaciones, que reportaron diferencias no estadísticamente significativas para MD y PSD y valores ligeramente inferiores para VFI (3). En la prueba de Hemicampo para Glaucoma (PHG) la mayoría de los resultados fueron clasificados como fuera de límites normales y las dos estrategias presentaron similares resultados en cada categoría. La mayoría de las evaluaciones obtuvieron el mismo resultado para PHG.

Ambas estrategias presentaron índices de confiabilidad dentro de los parámetros aceptables. Los falsos positivos fueron estadísticamente superiores para la estrategia SITA FASTER $(8,39 \pm 13,01)$ que para SITA ESTÁNDAR $(3,64 \pm 8,54)$, en la misma proporción a lo hallado por investigaciones similares. La Estrategia SITA Estándar usa un sesgo de inicio positivo, mostrando por lo tanto más estímulos que son ligeramente más sencillos de percibir. Una situación diferente se da con la estrategia SITA FASTER lo que influye como los pacientes definen su criterio de respuesta, son más propensos a responder cuando están más inseguros lo que de por sí debería incrementar las respuestas falso-positivas (11).

Los resultados generales indican que es posible reemplazar la estrategia SITA Estándar 24 -2 con la estrategia SITA FASTER 24 - 2. La discreta correspondencia para la prueba de hemicampo de glaucoma sugiere evaluaciones con la estrategia SITA Estándar 24 - 2 para pacientes con sospecha de glaucoma o estadios iniciales de Glaucoma Primario de Ángulo Abierto.

Este cambio será especialmente útil para aumentar la frecuencia de exámenes campimétricos en los servicios de oftalmología, con una Mayor frecuencia de exámenes es importante para detección más temprana 
de progresión del GPAA y para determinar la tasa de progresión y así mejorar las conductas terapéuticas que se toman con los pacientes que acuden a nuestro servicio.

\section{CONTRIBUCIÓN DE LOS AUTORES}

JMPB: ha contribuido en el seguimiento, recaudación de datos de los pacientes, realización de los estudios campímetros y análisis de datos. GV: ha contribuido en el seguimiento, recaudación de datos de los pacientes, realización de los estudios campímetros y análisis de datos. MA: ha sido el especialista en indicar el tratamiento a seguir y seguimiento del paciente, y conducción de los datos obtenidos. Todos los autores contribuyeron con la aprobación de la versión final del manuscrito.

\section{REFERENCIAS}

1. Skuta G, Cantor L, American Academy of Ophthalmology. Glaucoma. In: Basic and Clinical Science Course. 2017-2018 ed, pp. 61 - 84. Vol. 10. American Academy of Ophthalmology. 2018.

2. Bruce E. Prum Jr., MD, Lisa F. Rosenberg, American Academy of Ophthalmology Glaucoma Panel. Preferred Practice Pattern Guidelines. Primary Open-Angle Glaucoma. San Francisco: American Academy of Ophthalmology, 2010.

3. Silva L, Najafi A, Suwan Y, Teekhasaenee C, Ritch R. The iridocorneal endothelial syndrome. Survey of $\begin{array}{lll}\text { Ophthalmology } & \text {.2018;63 } & \text { (5):665-76. }\end{array}$ https://dx.doi.org/10.1016/j.survophthal.2018.01.001

4. Bengtsson B, Heijl A, Olsson J. Evaluation of a new threshold visual field strategy, SITA, in normal subjects: Evaluation of a new threshold visual field strategy, SITA, in normal subjects. Acta Ophthalmologica Scandinavica .1998;76 (2):165-9. $\quad$ https://dx.doi.org/10.1034/i.16000420.1998.760208.x.r

5. Johnson CA, Adams CW, Lewis RA. Fatigue effects in automated perimetry. Appl Opt.1988;27 (6):1030. https://dx.doi.org/10.1364/AO.27.001030

6. Jampel HD, Singh K, Lin SC, Chen TC, Francis BA, Hodapp E, et al. Assessment of Visual Function in Glaucoma. Ophthalmology $\quad .2011 ; 118 \quad$ (5):986-1002. https://dx.doi.org/10.1016/j.ophtha.2011.03.019

7. Bengtsson B, Heijl A. SITA Fast, a new rapid perimetric threshold test. Description of methods and evaluation in patients with manifest and suspect glaucoma: SITA Fast, a new rapid perimetric threshold test. Description of methods and evaluation in patients with manifest and suspect glaucoma. Acta Ophthalmologica Scandinavica $.1998 ; 76 \quad$ (4):431-7. https://dx.doi.org/10.1034/j.1600$\underline{0420.1998 .760408 . x}$

8. Malik R, Baker H, Russell RA, Crabb DP. A survey of attitudes of glaucoma subspecialists in England and Wales to visual field test intervals in relation to NICE guidelines. BMJ Open.2013;3 (5):e002067. https://dx.doi.org/10.1136/bmjopen-2012-002067

9. Artes PH, Iwase A, Ohno Y, Kitazawa Y, Chauhan BC. Properties of perimetric threshold estimates from Full Threshold, SITA Standard, and SITA Fast strategies. Invest Ophthalmol Vis Sci.2002;43 (8):2654-9. URL.

10. Centeno ED de, Hidalgo J, Quiroz M, Juárez R., Calibración de dos observadores para evaluar para evaluar restauraciones en una investigación clínica. Aplicación del Índice de Concordancia de KAPPA. 4, 2011, Vol. EFACIM, pp. 7 - 11.

11. Phu J, Khuu SK, Agar A, Kalloniatis M. Clinical Evaluation of Swedish Interactive Thresholding Algorithm-Faster Compared With Swedish Interactive Thresholding Algorithm-Standard in Normal Subjects, Glaucoma Suspects, and Patients With Glaucoma. American Journal of Ophthalmology.2019;208:251-64.

https://dx.doi.org/10.1016/j.ajo.2019.08.013 